\title{
Glypican-1 targeted antibody-based therapy induces preclinical antitumor activity against esophageal squamous cell carcinoma
}

\author{
Emi Harada ${ }^{1, *}$, Satoshi Serada ${ }^{1, *}$, Minoru Fujimoto ${ }^{1}$, Yusuke Takahashi', Tsuyoshi

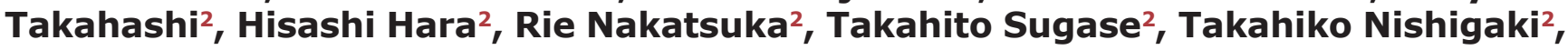 \\ Yurina Saito ${ }^{2}$, Kosuke Hiramatsu ${ }^{1}$, Satoshi Nojima ${ }^{3}$, Risa Mitsuo ${ }^{1}$, Tomoharu

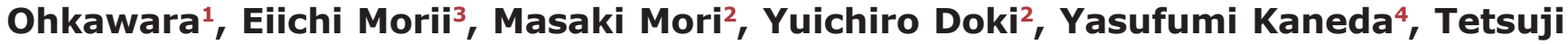 \\ Naka $^{1}$ \\ ${ }^{1}$ Laboratory of Immune Signal, National Institute of Biomedical Innovation, Health and Nutrition, Osaka, 567-0085, Japan \\ ${ }^{2}$ Department of Gastroenterological Surgery, Osaka University Graduate School of Medicine, Osaka, 565-0871, Japan \\ ${ }^{3}$ Department of Pathology, Osaka University Graduate School of Medicine, Osaka, 565-0871, Japan \\ ${ }^{4}$ Division of Gene Therapy Science, Osaka University Graduate School of Medicine, Osaka, 565-0871, Japan \\ *These authors contributed equally to this work and share first authorship \\ Correspondence to: Satoshi Serada, email: serada@nibiohn.go.jp \\ Tetsuji Naka, email: tnaka@nibiohn.go.jp
}

Keywords: esophageal squamous cell carcinoma, glypican-1

Received: August 17, 2016 Accepted: February 15, 2017 Published: March 01, 2017

Copyright: Harada et al. This is an open-access article distributed under the terms of the Creative Commons Attribution License (CC-BY), which permits unrestricted use, distribution, and reproduction in any medium, provided the original author and source are credited.

\section{ABSTRACT}

Esophageal squamous cell carcinoma (ESCC) has a poor prognosis despite the development of multimodal therapy. Expression of glypican-1 (GPC1) has been reported to be elevated in a subset of patients with ESCC and associated with chemoresistance. This study aimed to determine the association of GPC1 with ESCC growth and potential usefulness of the GPC1 targeted therapy by monoclonal antibody $(\mathrm{mAb})$ in ESCC. Expression of GPC1 was higher in ESCC tumor tissues than in adjacent non-tumoral tissues and normal tissues. Knockdown of GPC1 decreased growth of ESCC cells and induced apoptosis via inhibition of EGFR, AKT and p44/42-MAPK signaling pathways in vitro. Anti-GPC1 mAb strongly inhibited tumor growth via antibody-dependent cellular cytotoxicity dependent and independent manner in GPC1positive ESCC xenograft models. Anti-GPC1 mAb also inhibited tumor growth of GPC1 positive ESCC patients derived tumor xenograft models. Furthermore, anti-GPC1 mAb showed a significant tumor growth inhibition with decreased angiogenesis compared with IgG treated controls in ESCC xenografted mice. Treatment with anti-GPC1 mAb was not toxic in mice. Anti-GPC1 mAb may have a potent anti-tumor effect and represent a novel treatment option for patients with GPC1-positive ESCC.

\section{INTRODUCTION}

Esophageal cancer is the sixth leading cause of cancer death worldwide and esophageal squamous cell carcinoma (ESCC) is the predominant histological type in Japan and Eastern countries [1]. Despite the development of multimodal therapy comprising surgery, chemotherapy and radiotherapy, the prognosis of ESCC remains poor [2]. To improve unfavourable outcomes of ESCC, it is important to explore the molecular mechanisms underlying ESCC and allow the development of urgently required novel therapeutic strategies.
Molecular targeted agents have become front-line cancer therapies. Molecular targeted therapy can act on various molecular pathways, including those involved in growth factor receptor signaling [epidermal growth factor receptor (EGFR) and Her-2/neu], the cell cycle, apoptosis and angiogenesis. In breast carcinoma, targeted therapy against HER2 using the humanized monoclonal antibody trastuzumab has now become integrated into standard adjuvant treatment regimens and has led to significant improvements in disease-free and overall survival in patients with Her2-positive cancer [3]. Therefore, the identification of potential cancer antigens for the 
development of innovative cancer-targeted therapies has become essential.

Recently, our group has identified glypican-1 (GPC1) as a novel cancer antigen for ESCC by quantitative proteomic approach focused on cell surface membrane protein [4]. Expression of GPC1 was elevated in most patients with ESCC and high expression levels of GPC1 were significantly associated with poor prognosis as well as chemoresistance [4]. GPC1 is a member of the glypican family of heparan sulphate proteoglycans (HSPGs) that are bound to the cell surface of the plasma membrane via glycosylphosphatidylinositol (GPI) linkages [5]. Several heparin-binding growth factors (HBGFs), including heparin-binding EGF-like growth factor (HBEGF), fibroblast growth factor-2 (FGF-2) and hepatocyte growth factor (HGF) require HSPGs as co-receptors for efficient signalling [6]. GPC1 has been reported to enhance the interaction of several HBGFs with their specific receptors and modulate their biological activity [7]. Among these HBGFs, HB-EGF is a ligand of EGFR, is a member of the c-erb receptor family and is implicated in cell proliferation, differentiation and survival $[8,9]$. Moreover, over-expression of EGFR has been observed in $50 \%-70 \%$ of ESCC tumors and is associated with poor prognosis $[10,11]$.

Our present study demonstrated that increased expression of GPC1 was associated with ESCC cell growth and survival by partially enhancing EGFR activity to suppress apoptosis. In addition, we developed an antiGPC1 monoclonal antibody (mAb), which cross-reacts with mouse GPC1. Anti-GPC1 mAb induced significant tumor growth inhibition in ESCC xenograft models via antibody-dependent cellular cytotoxicity (ADCC) and complement-dependent cytotoxicity (CDC) dependent and independent manner. Importantly, anti-GPC1 mAb also induced potent tumor growth inhibition in GPC1 positive ESCC patient derived-tumor xenograft models. Furthermore, minimal toxicity was observed with antiGPC1 mAb treatment in mice. These results suggest GPC1 may be a promising targeted therapy for ESCC.

\section{RESULTS}

\section{Confirmatory expression analysis of GPC1 in human normal tissues and ESCC tumors}

We have reported that expression of GPC1 was elevated in most patients with ESCC [4]. To evaluate the specificity of the expression of GPC1, we analysed expression profile of GPC1 in various normal tissues at mRNA levels by real time PCR analysis. We found that the expression levels of GPC1 were relatively low compared to TE11 cells, while slight expression of GPC1 was observed in testis, ovary and heart (Figure 1A).

Next, expression of GPC1 in normal tissues was evaluated by immunohistochemical (IHC) analyses using normal tissue microarray. Although GPC1 was strongly expressed in testis, GPC1 was weakly expressed in heart, kidney, ovary, placenta, adrenal gland and thyroid (Figure 1B). GPC1 expression was very weak or undetectable in lung, liver, pancreas stomach, small intestine, colon prostate, thymus and brain (Figure 1B). By western blotting, expression levels of GPC1 in human normal heart, kidney, small intestine and colon were weak compared to ESCC tissues (Figure 1D). As previously reported [4], IHC staining of GPC1 in tissue sections from patients revealed intense GPC1 staining in ESCC compared with that in normal esophageal tissue (Figure 1C). In addition, IHC analyses showed membranous immunoreactivity in ESCC cells, indicating GPC1 was localized to the cell surface. However, expression of GPC1 in normal esophagus was weak compared to ESCC (Figure 1C). In ESCC, lymph node metastasis is known to be strongly associated with poor prognosis [12]. Intriguingly, expression of GPC1 was also detected in lymph node ESCC metastases, indicating GPC1 may represent a therapeutic target for ESCC with lymph node metastasis (Figure 1C). These data indicate GPC1 may be an attractive therapeutic target for ESCC therapy.

\section{Knockdown of GPC1 expression induces growth inhibition of ESCC cells in vitro}

To examine whether GPC1 expression contributes to growth of ESCC cells, the effect of GPC1-siRNA treatment in two ESCC cell lines expressing GPC1 (TE8 and TE14) as previously reported [4], was evaluated using the WST-8 assay. To ensure silencing efficiency, decreased expression of GPC1 was analysed by Western blot analysis $48 \mathrm{~h}$ after siRNA transfection (Figure 2A). There was marked inhibition of cell growth in GPC1siRNA-transfected cells compared with negative control (NC)-siRNA-treated cells and untreated cells in each ESCC cell line (Figure 2B). In TE8 and TE14 cells treated with GPC1 siRNA, markedly increased caspase- 3 activity was detected (Figure 2C). In addition, knockdown of GPC1 in TE8 and TE14 cells decreased levels of the antiapoptotic protein Bcl-w, and increased levels of the proapoptotic proteins Bim in TE8 cells and Bak in TE14 cells (Figure 2D), suggesting that suppression of GPC1 protein expression by siRNA resulted in increased apoptosis in these ESCC cell lines.

\section{Knockdown of GPC1 expression inhibits EGFR, AKT and p44/42-MAPK signalling pathways in ESCC cells in vitro}

Previously, it has been reported that there is an association between EGFR signalling and ESCC progression, and several types of EGFR targeted therapy were recently developed $[13,14]$. GPC1 has been shown to be a co-receptor of various HBGFs, including HB-EGF, 
amphiregulin, HRG and FGF-2, which promote biological activity [15]. To elucidate proliferation-associated signalling pathways regulated by GPC1 in ESCC cells, phosphorylation levels of EGFR were analysed by Western blot analysis. Notably, phosphorylation levels of EGFR (Tyr1068) were decreased in cells transfected with GPC1 siRNA compared with NC-siRNA-treated cells and untreated cells (Figure 2E). AKT and p44/42-MAPK signalling pathways are major downstream effectors of the EGFR signalling pathway associated with survival and proliferation of cancer cells. In accordance with the inhibition of phospho-EGFR (Tyr1068), knockdown of GPC1 also decreased levels of phospho-AKT (Thr308), phospho-p70S6K (Thr389) and phospho-p44/42-MAPK (Thr202/Try204) in TE-8 cells (Figure 2E). In addition, treatment with the MEK1 inhibitor PD98059 resulted in increased expression of Bim and decreased expression of Bcl-w (Figure 2F) or the PI3 kinase inhibitor Ly294002 resulted in decreased expression of Bcl-w (Figure 2F), consistent with results obtained from GPC1 siRNA transfection studies.

\section{Knockdown of GPC1 expression inhibits activation of EGFR via HB-EGF in ESCC cells in vitro}

We assessed the effects of the GPC1 knockdown on the activation of EGFR by stimulation with HB-EGF. Compared with the control siRNA-transfected TE8 cells, knockdown of GPC1 resulted in decreased activation

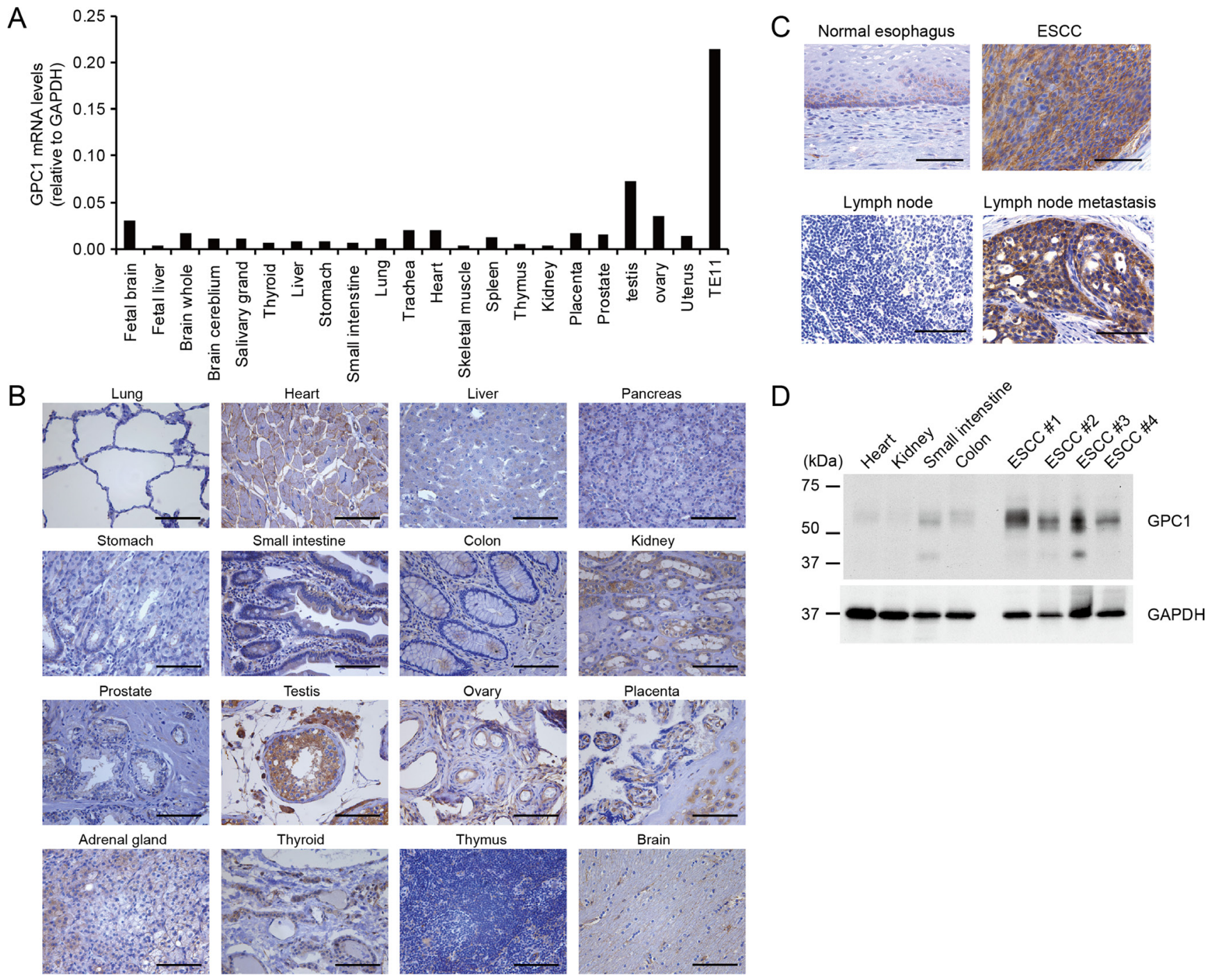

Figure 1: Confirmatory expression analysis of GPC1 in human normal tissues and ESCC tumors. (A) Quantitative realtime PCR analyses were used to quantify GPC1 mRNA in various normal human tissues and GPC1 positive TE11 cells; GAPDH was used as an internal control. (B) Representative IHC GPC1 staining in normal tissues. Scale bar, $100 \mu \mathrm{m}$. (C) Representative IHC GPC1 staining in primary ESCC tissues, lymph node metastasis in ESCC and esophageal adenocarcinoma. Scale bar, $100 \mu \mathrm{m}$. (D) Western blot analysis of GPC1 in normal human heart, kidney, small intestine and ESCC tumors. Western blotting with anti-GPC1 antibody against proteins treated with heparinase III. 
of phospho-EGFR (Tyr1068) following stimulation with HB-EGF (Figure 2G left panel). In addition, AKT phosphorylation in response to stimulation with HB-EGF was attenuated by transfection with GPC1 siRNA compared with NC siRNA in transfected TE8 cells (Figure 2G right panel), suggesting that GPC1 regulates EGFR activity and downstream AKT signalling induced by HB-EGF.

\section{Production of anti-GPC1 mAb}

Because GPC1 is frequently over-expressed in ESCC and associated with increased growth in ESCC, we attempted to develop an antibody-based therapy targeting GPC1. Because hGPC1 and mouse GPC1 (mGPC1) proteins are highly homologous $(88.71 \%$ sequence identity), hGPC1 likely has little antigenicity in mice. We therefore used chickens as hosts for antigen immunization. Single-chain variable fragment $(\mathrm{scFv})$ clones positively bound to GPC1 were selected and chicken/mouse chimeric $\mathrm{mAb}$ with mouse $\operatorname{IgG} 2 \mathrm{a} \mathrm{Fc}$ domains were generated as mouse IgG2a mediates high levels of ADCC and CDC activity [16]. We then tested the affinity of the chicken/ mouse chimeric anti-GPC1 $\mathrm{mAb}$ in two native ESCC cell lines (TE8 and TE14), one GPC1-negative lung squamous carcinoma cell line (LK2) and an LK2-derived cell line (LK2-hGPC1) by flow cytometry. The LK2hGPC1 cell line expresses stable and high levels of cell surface GPC1. Chicken/mouse chimeric anti-GPC1 mAb (clone 1-12) demonstrated specific binding to ESCC cells and LK2-hGPC1 cells but not to GPC1-negative LK2 cells (Figure $3 \mathrm{~A}$ ). We measured the binding affinities of chicken/mouse chimeric anti-GPC1 $\mathrm{mAb}$ to GPC1 protein by SPR analysis. The calculated $\mathrm{K}_{\mathrm{D}}$ value was $2.61 \mathrm{nM}$ for clone 1-12, comparable with the affinities of approved cancer therapeutic antibodies (Figure 3B).

\section{Epitope mapping, cross-reactivity and indirect cytotoxity assay of anti-GPC1 mAb}

Mass spectrometry was used to determine the epitope region of the anti-GPC1 $\mathrm{mAb}$. hGPC1 protein was incubated with anti-GPC1 mAb or control mouse IgG2a
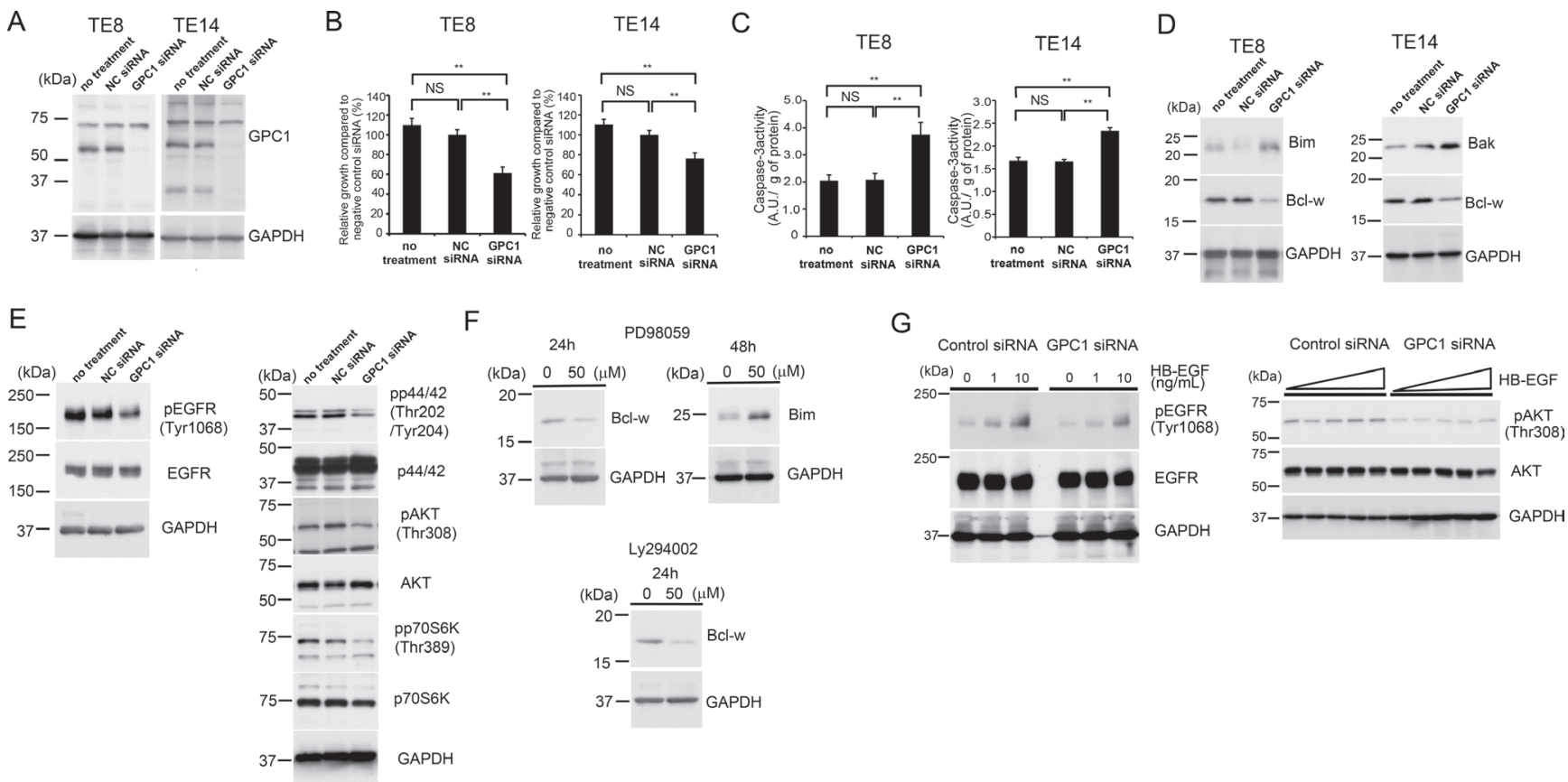

Figure 2: GPC1 is associated with ESCC proliferation by regulating AKT, p44/42 and EGFR signalling pathways. (A) Knockdown of GPC1 expression by transfection with GPC1 siRNA. TE8 and TE14 cells were transfected with GPC1 or NC siRNA. Forty eight hours after transfection, knockdown of GPC1 was confirmed by Western blot analysis. (B) TE8 and TE14 cells were transfected with siRNA. Cell growth was assessed at $120 \mathrm{~h}$ using the WST-8 assay. Values were normalized to NC-siRNA-treated cells. (C) Caspase-3 activities were measured $72 \mathrm{~h}$ after siRNA transfection. (D) Knockdown of GPC1 increased expression of pro-apoptotic proteins and decreased expression of anti-apoptotic proteins. TE8 and TE14 cells were transfected with GPC1 siRNA or NC siRNA for 48 h. Extracted proteins were immunoblotted with indicated antibodies. (E) Constitutive activation of EGFR, AKT and p44/42-MAPK signalling pathways was inhibited by knockdown of GPC1. TE8 cells were transfected with GPC1 siRNA or NC siRNA. After 48 h, protein extracts were immunoblotted with indicated antibodies. (F) Increased expression of pro-apoptotic proteins and decreased expression of anti-apoptotic proteins was observed after treatment with PD98059 or Ly294002 for 24 or 48 h in TE8 cells. Protein extracts were immunoblotted with indicated antibodies. (G) GPC1 enhances activation of EGFR by HB-EGF. TE8 cells were transfected with GPC1 siRNA or NC siRNA for $48 \mathrm{~h}$. (Left panel) Cells were stimulated with 0, 1.0, $10 \mathrm{ng} / \mathrm{mL}$ HB-EGF for $10 \mathrm{~min}$. (Right panel) After serum starvation for $3 \mathrm{~h}$, cells were stimulated with $0,1.25,2.5,5,10 \mathrm{ng} / \mathrm{mL}$ HB-EGF for $15 \mathrm{~min}$. Protein extracts were immunoblotted with indicated antibodies. 
and digested with trypsin. After immunoprecipitation with protein G-Sepharose, antibody-bound peptides were eluted and amino acid regions were identified by LC-MS/ MS analysis. GPC1-derived peptides 339-358, 388-404 and 405-421 were specifically identified as anti-GPC1 $\mathrm{mAb}$-bound peptide compared with control mouse IgG2a (Figure 3C).

To determine whether anti-GPC1 mAb cross-reacted with mGPC1, HEK293 cells were transfected with an mGPC1 expression vector. In addition to showing affinity for hGPC1, we found anti-GPC1 $\mathrm{mAb}$ also reacted with mGPC1 by flow cytometry (Figure 3D).

\section{Toxicology of anti-GPC1 mAb}

Because anti-GPC1 mAb cross-reacted with mGPC1, in vivo toxicology studies were performed in $\mathrm{C} 57 \mathrm{BL} / 6$ mice to examine the toxicity of anti-GPC1 mAb. Treatment with anti-GPC1 $\mathrm{mAb}$ antibody at a dose of $50 \mathrm{mg} / \mathrm{kg}$ did not cause significant changes in serum chemistry or blood cell counts after 7 days compared with mice treated with IgG2a control antibodies (Supplementary Tables 1, 2). No histologic changes in liver, lung, heart, kidney, spleen, brain and testis were observed following treatment with anti-GPC1 mAb (Supplementary Figure 1).

\section{Anti-GPC1 mAb induces anti-tumor activity in vivo}

To evaluate the antitumor activity of anti-GPC1 $\mathrm{mAb}$ in animals, SCID mice were subcutaneously inoculated with TE14 cells and then intraperitoneally treated with $10 \mathrm{mg} / \mathrm{kg}$ anti-GPC1 mAb twice-weekly for three weeks. Compared with isotype control mouse IgG2a, administration of anti-GPC1 mAb significantly inhibited the growth of the TE14 xenografts assessed by tumor volume $(60.99 \% \pm 5.11 \%$ tumor growth inhibition at day 32$)$ and tumor weight (Figure 4A).

Next, we also assessed the anti-tumor effect of antiGPC1 $\mathrm{mAb}$ against ESCC patient tumor-derived xenograft (PDX) model (designated ESCC-8). ESCC tissues were subcutaneously implanted to the NOG mice. Furthermore, tumor tissues were subcutaneously implanted to the SCID mice to assess the anti-tumor effect of anti-GPC1 mAb. In this ESCC-8 PDX model, expression of GPC1 in the tumor tissue was confirmed by IHC analysis (Supplementary Figure 2). Anti-GPC1 mAb significantly inhibited the growth of the ESCC-8 PDX compared with isotype control mouse IgG2a $(67.87 \% \pm 6.28 \%$ tumor growth inhibition at day 31 ) and also tumor weight (Figure 4B). By TUNEL staining, we detected marked apotosis in tumors of antiGPC1 mAb treated mice compared to control IgG treated mice in both TE14 xenograft and ESCC-8 PDX models (Figure 4C and 4D).

NOD/SCID mice, known to have low natural killer cell activity and no $\mathrm{CDC}$ activity and functional $\mathrm{B}$ and $\mathrm{T}$ cells, were used to assess the contribution of ADCC and $\mathrm{CDC}$ to the anti-tumor effect of anti-GPC1 mAb. Notably, while anti-GPC1 mAb seemed less effective in NOD/SCID mice than in SCID mice, it still showed tumor growth inhibition $(38.7 \% \pm 6.39)$ in TE14 xenografts in NOD/SCID mice compared with control mice treated
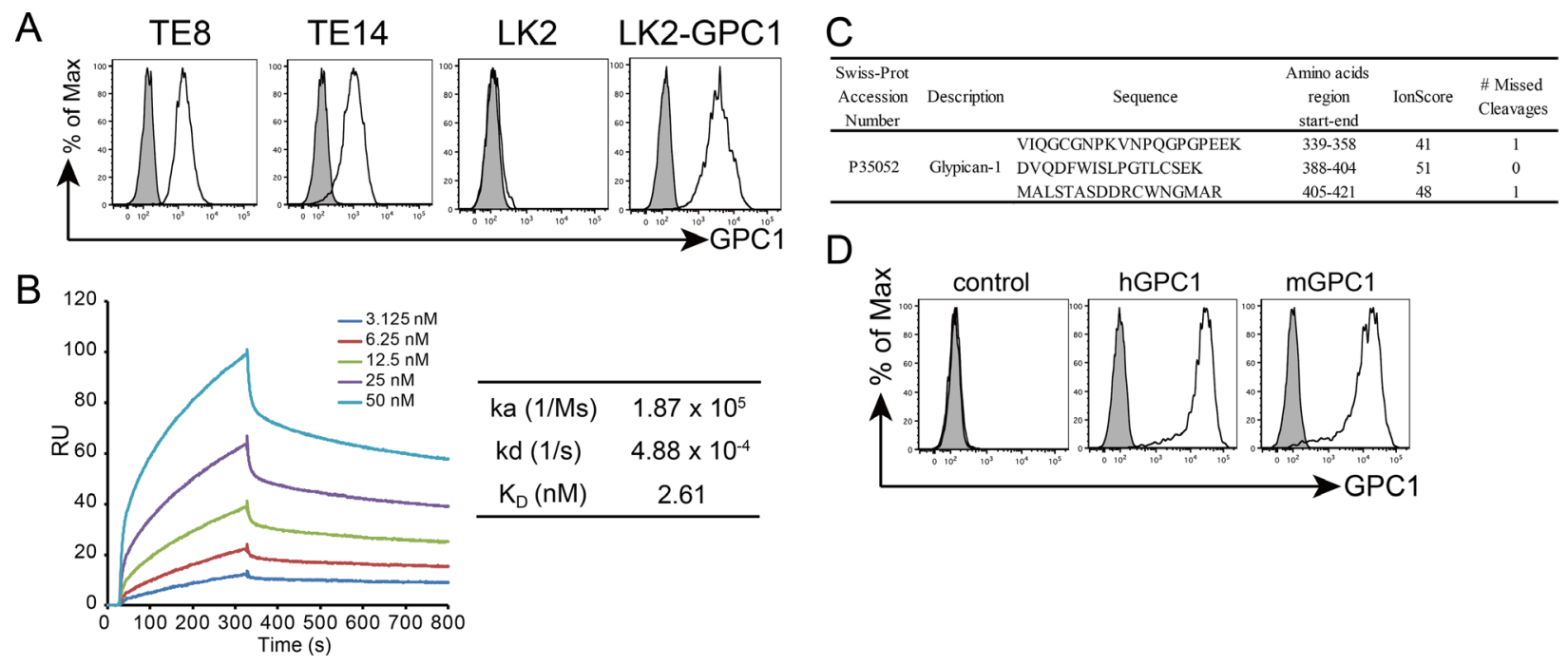

D

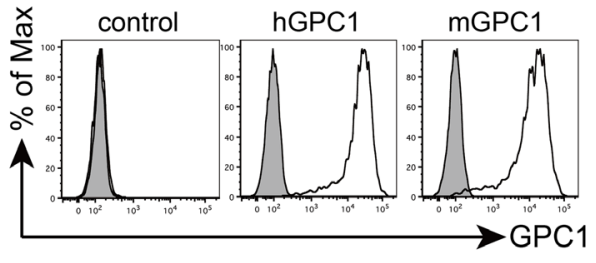

Figure 3: Isolation and characteristics of human antibody targeting GPC1. (A) Flow cytometry of antibody binding to GPC1positive (TE8, TE14), GPC1-negative (LK2) and cells stably transfected with the LK2 gene encoding GPC1 (LK2-GPC1). The shaded area histogram profile indicates the isotype control. Open histogram indicates anti-GPC1 mAb staining. (B) SPR analysis of anti-GPC1 mAb. (C) Epitope analysis of anti-GPC1 mAb by mass spectrometry. Recombinant human GPC1 proteins were mixed with anti-GPC1 mAb or mouse IgG2a and digested with trypsin. After digestion, immune complexes were immunoprecipitated with protein G-Sepharose. Eluted peptides were reduced, alkylated and analyzed by LC-MS/MS. (D) Anti-GPC1 mAb clone 1-12 cross-reacted with mGPC1. HEK293 cells were transfected with empty vector, full length hGPC1 or mGPC1 expression vectors. The shaded histogram profile indicates control samples without primary antibody staining and the open histogram indicates results of anti-GPC1 antibody staining. 
with mouse IgG2a (Figure 5A). We found that GPC1 was expressed in vascular endothelium cells in ESCC tumor (Supplementary Figure 3), and tumor angiogenesis might be inhibited by anti-GPC1 $\mathrm{mAb}$ in vivo. By CD31 staining, we observed significantly decreased blood vessels in anti-GPC1 mAb treated mice compared to control IgG2a (Figure 5B). These results suggest that anti-GPC1 mAb inhibits tumor growth in vivo in ADCC and $\mathrm{CDC}$ dependent and independent manners including inhibition of tumor angiogenesis.

\section{DISCUSSION}

In this study, we demonstrated GPC1 as a suitable ESCC cancer antigen for antibody-based therapy. GPC1 was associated with increased proliferation of ESCC through inhibition of apoptosis (Figure 2). GPC1 was also found to be involved in the activation of the EGFR signalling pathway in response to EGFR ligands including HB-EGF, which is known to be one of the HBGFs. Importantly, we have recently demonstrated that high tumoral expression of GPC1 in ESCC, determined by IHC analysis, was significantly associated with poor prognosis compared to low expression of GPC1 and higher expression of GPC1 associated with elevated chemoresistance to cisplatin. We also demonstrated that anti-GPC1 mAb (clone 1-12) induced marked tumor growth inhibition in GPC1-positive human ESCC xenograft models. Considering that the expression of GPC1 in normal tissue is limited, targeting GPC1 by anti-GPC1 $\mathrm{mAb}$ would be a promising therapy for ESCC expressing GPC1.

It has previously been reported that GPC1 is involved in cell cycle regulation [17-19]. Qiao et al. demonstrated ectopic expression of GPC1 stimulates S phase entry via downregulation of tumor suppressors, including $\mathrm{pRb}$ and $\mathrm{Cip} / \mathrm{Kip}$ cyclin-dependent kinase inhibitors, and upregulation of pro-oncogenic proteins, including cyclin E and cyclin-dependent kinase 2, in the human glioblastoma cell line U87-MG [19]. Silencing of GPC1 expression by siRNA transfection induced G0/ G1 growth arrest in TE8 and TE14 cells (Supplementary Figure 4). However, the role of GPC1 in the regulation of apoptosis has not previously been reported. As shown in Figure 2C, siRNA-mediated knockdown of GPC1 resulted in a significant level of apoptosis in TE8 and TE14 cells via induction of increased expression of the pro-apoptotic proteins Bim and Bik and decreased expression of the antiapoptotic protein Bcl-w. These data indicate a critical role of GPC1 in the growth and survival of ESCC cells through regulation of apoptosis in addition to the cell cycle.

We produced chicken/mouse chimeric mAb against human GPC1, which cross-reacts with mGPC1, and demonstrated marked tumor growth inhibition by antiGPC1 mAb in SCID mice xenografted with GPC1-positive TE14 cells via ADCC and CDC activity. Intriguingly,
anti-GPC1 $\mathrm{mAb}$ also represented potent antitumor effect against GPC1 positive ESCC PDX model, suggesting that the potential usefulness of this $\mathrm{mAb}$ in patients with GPC1 positive ESCC. In addition, anti-GPC1 $\mathrm{mAb}$ also partially inhibited tumor growth in NOD/SCID mice, inhibiting tumor angiogenesis (Figure 5B), suggesting that the possibility of the presence of neutralizing activity of GPC1 in this clone. Notably, GPC1 has previously been reported to be expressed on tumor vascular endothelial cells but not on normal vascular endothelial cells [20]. Furthermore, it has been reported that host-derived GPC1 in addition to tumoral GPC1 is involved in tumor neovascularization [21]. Considering that vascular endothelial growth factor and basic fibroblast growth factor, both of which are known as HBGFs, are important factors for the proliferation of vascular endothelial cells, anti-GPC1 mAb may inhibit tumor growth in vivo by blocking GPC1 as a co-receptor activity of these factors against vascular endothelial cells and inhibiting neovascularization, although further studies are required to completely elucidate this potential mechanism.

In our previous study [4], by IHC immunohistochemical assessment of 175 ESCC specimens, $98.8 \%$ expressed GPC1. We further showed that high GPC1 expression is associated with poor prognosis by KaplanMeier survival analysis and increased chemo-resistance by clinicopathological analysis. In addition, we also showed that GPC1 expression in vitro enhances chemo-resistance of ESCC to cisplatin not by increasing transport and excretion of drugs but by antagonizing apoptosis through upregulating MAPK signaling and Bcl-2 family signaling. These results indicate that GPC1 positive tumor cells are less sensitive to cisplatin. Since anti-GPC1 mAb specifically target GPC1 positive tumor cells, we consider that anti-GPC1 mAb would be quite effective therapy for treatment of ESCC cells which remain after cisplatin therapy.

The amino acid sequences of epitope regions recognized by anti-GPC1 $\mathrm{mAb}$ (clone 1-12) were highly conserved in hGPC1 and mGPC1. Because anti-GPC1 $\mathrm{mAb}$ cross-reacts with $\mathrm{mGPC} 1$, we assessed toxicity in mice treated with anti-GPC1 $\mathrm{mAb}$ and found no evidence of blood biochemistry changes or histological abnormalities, including esophagus and testis, demonstrating the low toxicity and therapeutic potential of anti-GPC1 mAb. We observed over-expression of GPC1 in primary ESCC tissue (Figure 1B), whereas expression of GPC1 was limited in other tissues such as the testis. Moreover, GPC1 was found to be expressed in lymph node metastases (Figure 1B), suggesting that systemic treatment with anti-GPC1 mAb may also have efficacy in patients with lymph node ESCC metastases. Further analyses are warranted regarding distant metastasis because the expression of GPC1 in distant metastasis was not examined in this study due to the difficulty in obtaining clinical samples. In addition to ESCC, increased expression of GPC1 has been reported in pancreatic cancer, breast cancer and glioma and shown 
to promote the mitogenic, metastatic and angiogenic properties of cancers [6, 21-24]. Therefore, anti-GPC1 $\mathrm{mAb}$ may have anti-tumor efficacy in these GPC1-positive cancers. We are currently conducting studies evaluating the anti-tumor efficacy of anti-GPC1 mAb in xenograft models using cancer types other than ESCC.
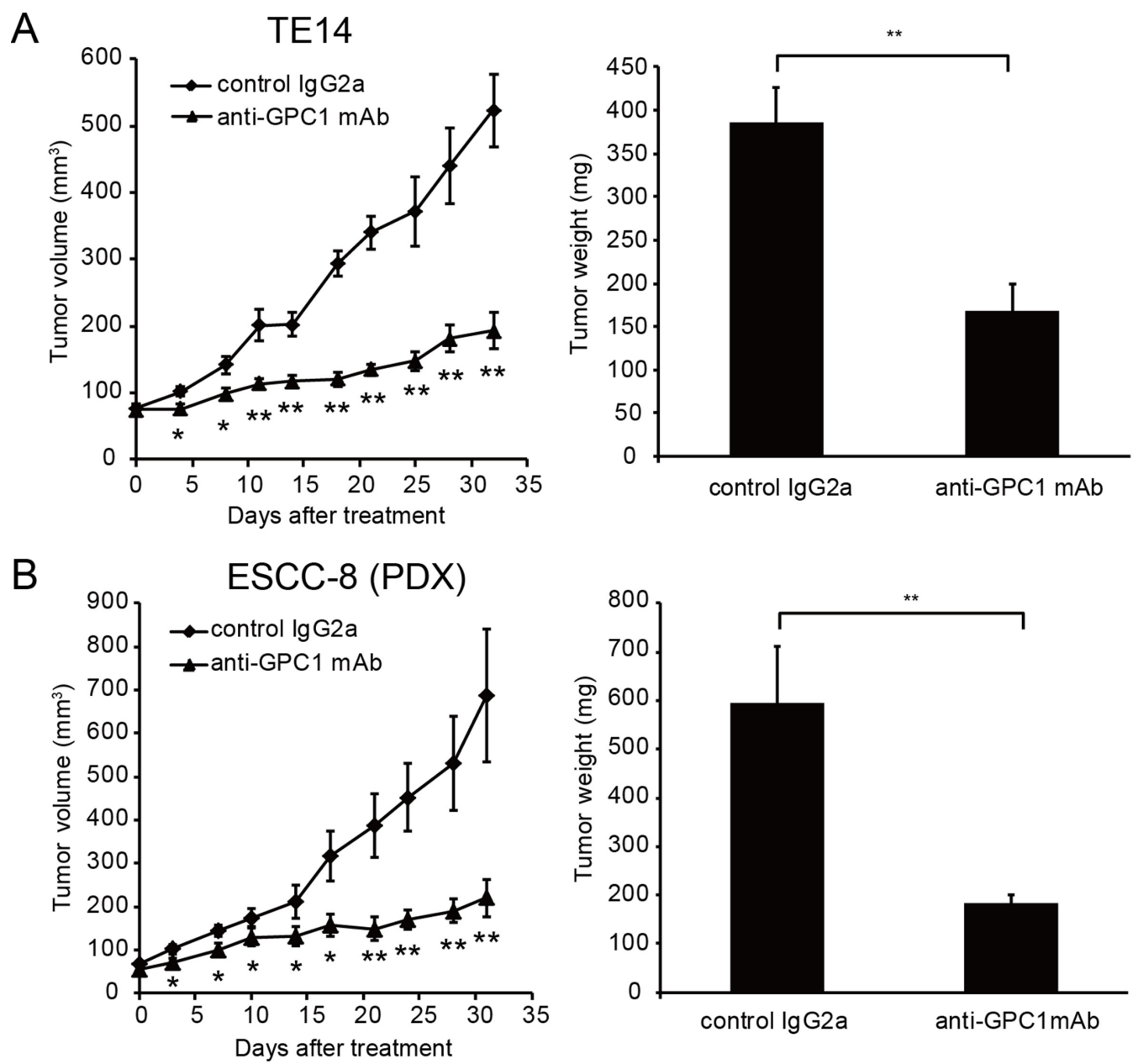

C

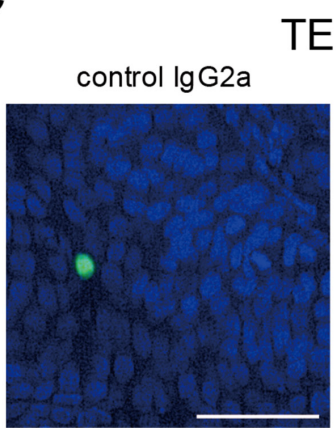

TE14
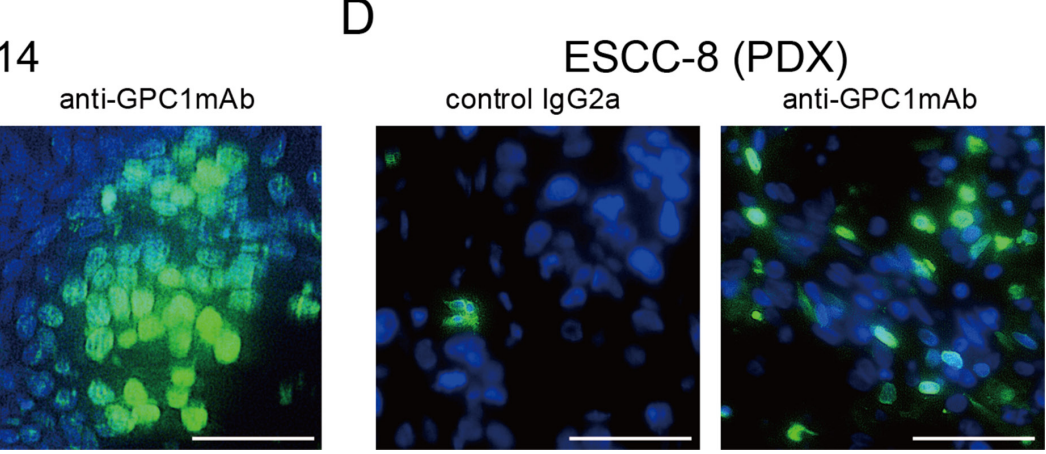

Figure 4: Anti-GPC1 mAb inhibits ESCC growth in SCID mice xenografted with GPC1 positive ESCC cell line and ESCC tissues in vivo. (A) Anti-tumor activity of anti-GPC1 mAb against TE14 xenografts. SCID mice were subcutaneously inoculated with $2 \times 10^{6}$ cells. Mice were xenografted with GPC1-positive (TE14) and divided into two groups (5-6 mice/group) when tumor volumes reached an average size of $70 \mathrm{~mm}^{3}$. Mice were intraperitoneally treated with $10 \mathrm{mg} / \mathrm{kg}$ anti-GPC1 mAb or control mouse IgG2a. Thirty two days (TE14 cells) after first treatment, tumors were removed and weighed. (B) Anti-tumor activity of anti-GPC1 mAb against ESCC8 patients tumor xenografted mice. SCID mice were subcutaneously inoculated with ESCC- 8 tumor tissues. Mice were divided into two groups ( 7 mice/group) when tumor volumes reached an average size of $70 \mathrm{~mm}^{3}$, and mice were intraperitoneally treated with $10 \mathrm{mg} / \mathrm{kg}$ anti-GPC1 mAb or control mouse IgG2a. Thirty one days after first treatment, tumors were removed and weighed. $* P<0.05$, $* * P<0.01$. $(\mathbf{C}, \mathbf{D})$ TUNEL assay (blue fluorescence $=$ DAPI staining for nuclei; cyan fluorescence $=$ TUNEL positivity) in TE14 (C) or ESCC-8 PDX tissues from animals treated with control mouse IgG2a or anti-GPC1 $\mathrm{mAb}$. Scale bar $=50 \mathrm{~mm}$. 
In conclusion, anti-GPC1 $\mathrm{mAb}$ inhibits ESCC growth by targeted inhibition of GPC1. Our data suggest that GPC1 is associated with tumor growth and that targeting GPC1 with anti-GPC1 mAb represents a therapeutic strategy to decrease tumor growth in patients with GPC1-positive ESCC.

\section{MATERIALS AND METHODS}

\section{Tissue samples}

Written informed consent was obtained for all cases and the experimental protocol was approved by the ethics committees of Osaka University and National Institute of Biomedical Innovation, Health and Nutrition.

\section{Cell lines}

Human esophageal squamous cancer cell lines (TE8, TE11 and TE14) were obtained from the RIKEN BioResource Center. A human lung squamous cancer cell line (LK2) was obtained from the Japanese Collection of Research Bioresources (Osaka, Japan). These cell lines were cultured in RPMI 1640 medium (Wako Pure Chemical Industries) supplemented with 10\% FBS (Serum Source International, NC, USA), $100 \mathrm{U} / \mathrm{ml}$ penicillin and $100 \mu \mathrm{g} / \mathrm{ml}$ streptomycin (Nacalai Tesque, Kyoto, Japan). Establishment of GPC1 stable transfectant LK2 cell lines were described previously [4], and cell lines were cultured in RPMI 1640 medium supplemented with 10\% FBS and $250 \mu \mathrm{g} / \mathrm{ml}$ G418 (Life technologies, Carlsbad, CA,USA). These cell lines were cultured at $37^{\circ} \mathrm{C}$ under a humidified atmosphere of $5 \% \mathrm{CO}_{2}$. The identity of each cell line was confirmed by DNA fingerprinting via short tandem repeat profiling, as previously described [25].

\section{Reagents and antibodies}

PD98059 and Ly294002 were purchased from Cell Signaling Technology (Danvers, MA). Recombinant human HB-EGF was purchased from R\&D Systems (Minneapolis, MN). The following primary antibodies were used: anti-GPC1 antibody from Atlas antibodies (Stockholm, Sweden); anti-phospho-Akt (Thr308), anti-
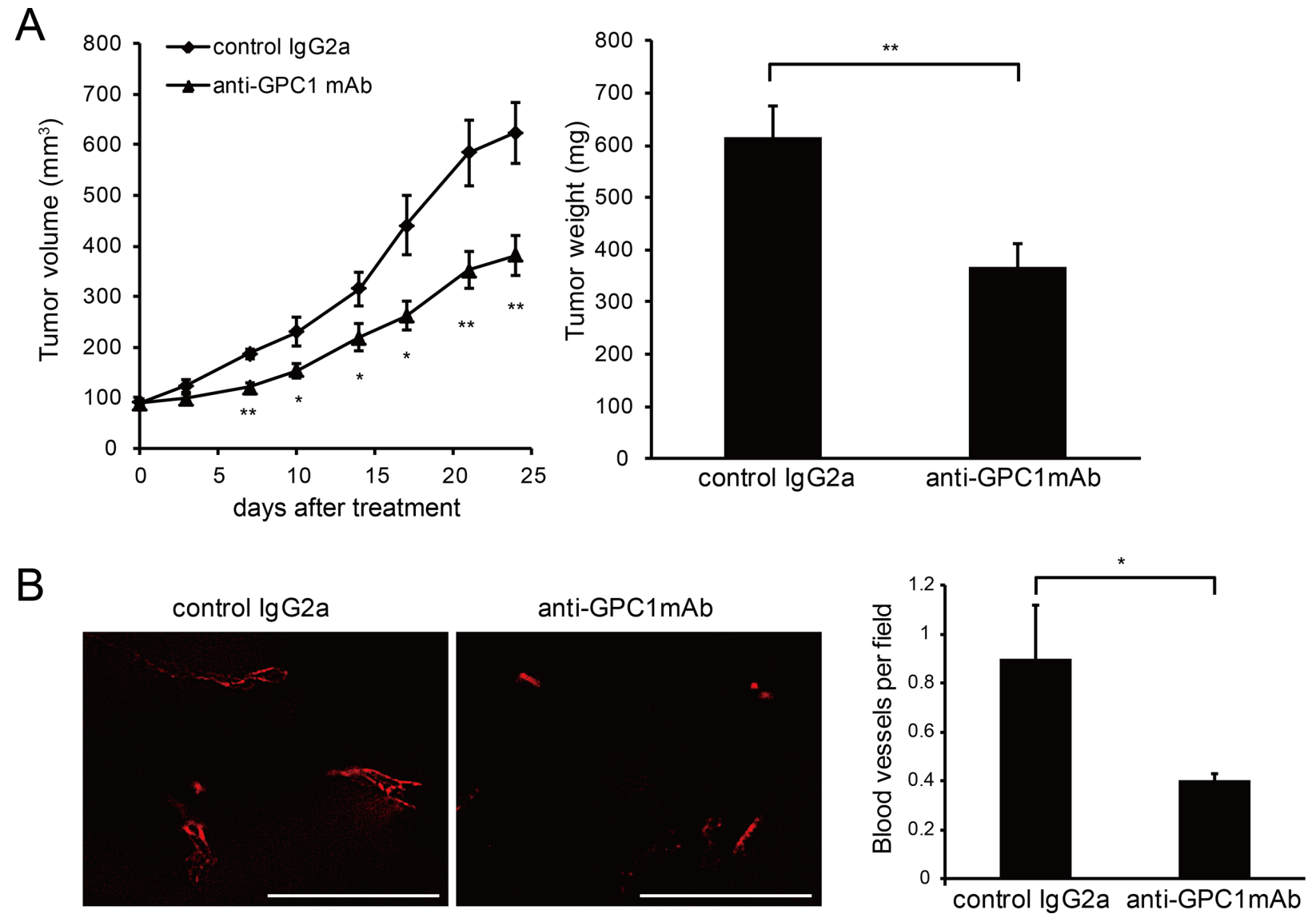

Figure 5: Anti-GPC1 mAb inhibits ESCC growth in NOD/SCID mice in vivo. Anti-tumor activity of anti-GPC1 mAb against TE14 in NOD/SCID mice. (A) NOD/SCID mice were subcutaneously inoculated with $2 \times 10^{6} \mathrm{TE} 14$ cells. When tumor volumes reached an average size of $70 \mathrm{~mm}^{3}$, mice were intraperitoneally treated with $10 \mathrm{mg} / \mathrm{kg}$ anti-GPC1 mAb or control mouse IgG2a. Twenty four days after first treatment, tumors were removed and weighed. (B) Immunohistochemical staining of CD31 (red) in TE14 xenografts NOD/SCID mice and quantification of CD31 positive area. Scale bar, $100 \mu \mathrm{m} . * P<0.05, * * P<0.01$. 
Akt, anti-phospho-p70S6K (Thr389), anti-p70S6K, antiphospho-p44/42 (Thr202/Tyr204), anti-p44/42, anti-Bak, anti-Bim, anti-Bcl-w and anti-phospho-EGFR (Tyr1068) from Cell Signaling Technology; anti-GAPDH from Santa Cruz Biotechnology (Santa Cruz, CA) and anti-EGFR from BD Transduction Laboratories (San Jose, CA).

\section{Quantitative reverse transcription-PCR (qRT- PCR) analysis}

Human MTC Panel I and Human MTC Panel II (Clontech, Palo Alto, CA, USA) were used as a source cDNA from several normal human tissues. For positive control total RNA was extracted from TE11 cells and cDNA was prepared as described previously [25]. To confirm expression of GPC1, qRT-PCR was performed as previously described [25]. GAPDH was used as a housekeeping gene for quantitative real-time PCR normalization. Primer sequences used were as follows: GPC1, forward primer 5'-GCCAGATCTACGG AGCCAAG-3' and reverse primer 5'-AGGTTCTCCTCC ATCTCGCT-3' and GAPDH, forward primer 5'- AGCA ATGCCTCCTGCACCACCAAC-3' and reverse primer 5'- CCGGAGGGGCCATCCACAGTCT-3'.

\section{Digestion with heparinase III}

Proteins were extracted from fresh-frozen samples of ESCC with $50 \mathrm{mM}$ Tris- $\mathrm{HCl}$ (pH 7.4), $150 \mathrm{mM} \mathrm{NaCl}$, $1 \mathrm{mM}$ EDTA, 1\% Triton X-100, 1\% protease inhibitor cocktail (Nacalai Tesque) and 1\% phosphatase-inhibitor cocktail (Nacalai Tesque). The total proteins from adult human normal heart, kidney, small intestine, colon tissues were purchased from BioChain Institute, Inc (Hayward,CA, USA). Protein extracts from ESCC tissues or normal tissues were digested with Heparinase III, as described previously [6], and used for western blotting.

\section{Western blotting}

Cells were lysed in radioimmunoprecipitation assay buffer (10 mM Tris-HCl, pH 7.5, $150 \mathrm{mM} \mathrm{NaCl,} \mathrm{1 \%}$ Nonidet P-40, 0.5\% sodium deoxycholate, $0.1 \%$ SDS, $1 \%$ protease-inhibitor cocktail and $1 \%$ phosphatase-inhibitor cocktail). Following centrifugation $\left(13,200 \mathrm{rpm}, 4^{\circ} \mathrm{C}\right.$, $15 \mathrm{~min}$ ), soluble proteins in the supernatant were separated using SDS-PAGE, as previously described [26].

\section{Immunohistochemistry}

Surgically resected tumor tissues were obtained from patients with ESCC from Osaka University Hospital (Osaka, Japan). Detailed methods are described in the online Supplementary Material.

Frozen sections were prepared from tumor tissues and stained for CD31 using a rat anti-mouse CD31 (BD
Biosciences) followed by the Alexa-647-conjugated second antibody. Fluorescence images were captured using Biozero BZ-9000 (Keyence, Tokyo, Japan) in five random fields at $400 \times$ magnification. The fluorescence was quantitated by a standardized procedure using a BZ-II Analyser (Keyence).

\section{Small interfering RNA transfection}

Commercial human GPC1 (hGPC1) small interfering RNA (siRNA) and negative control siRNA were obtained from QIAGEN (Valencia, CA, USA). Cells were transfected with siRNA using Lipofectamine 2000 reagent (Invitrogen), according to the manufacturer's instructions. For gene silencing, a specific sense strand (5'-gggacacgcucacggccaatt-3') was used for hGPC1 siRNA, and an antisense strand (5'-uuggecgugagcguguccetg-3') was used as a control. Selective silencing of hGPC1 was confirmed by Western blot analysis.

\section{Cell growth assay}

ESCC cells were plated in 96-well plates (1,000 cells/well for TE8 or 2,000 cells/well for TE14) and grown in their respective media for $120 \mathrm{~h}$ after siRNA transfection. At each time point, cell growth was assessed using the WST-8 assay as previously described [25].

\section{Apoptosis assay}

ESCC cells were seeded in 6-well plates at a density of $2 \times 10^{5}$ cells per well, and transfected with siRNA targeting hGPC1 or NC siRNA for $72 \mathrm{~h}$. The cells were washed with PBS, and caspase-3 activity was detected using the caspase-3 fluorometric assay kit (R\&D systems) according to the manufacturer's instructions. The presented values are representing the means of three independent experiments.

\section{Antibody production, epitope and cross- reactivity analysis of anti-GPC1 $\mathrm{mAb}$}

Detailed methods are described in the online Supplementary Material.

\section{Fluorescence activated cell sorting (FACS) analysis}

Cells were washed twice in PBS (Nacalai Tesque) and detached with $0.02 \%$ EDTA solution (Nacalai Tesque). Cells were washed twice with cold FACS buffer (PBS supplemented with $1 \%$ FBS and $0.1 \%$ sodium azide) and then incubated with chicken/mouse chimeric anti-GPC1 antibody (clone 1-12) at a 1:100 dilution and labelled with FITC-labelled goat anti-mouse $\operatorname{IgG}(\mathrm{H}+\mathrm{L}$ chain specific) antibody (SouthernBiotech, Birmingham, AL, USA). Stained cells were analysed using a FACS Canto II 
cytometer (Becton Dickinson, Mountain View, CA, USA) and the results were analysed using FlowJo software (Tree Star, Stanford, CA, USA).

\section{Surface plasmon resonance (SPR) analysis}

The binding affinity of each anti-hGPC1 antibody to hGPC1 was assessed by SPR using BIAcore3000 (GE Healthcare UK Ltd., Chalfont, United Kingdom). Detailed methods are described in the online Supplementary Material.

\section{In vivo toxicology studies}

$\mathrm{C} 57 \mathrm{~B} / 6$ mice at 8 weeks were administered with anti-GPC1 mAb (i.p. $50 \mathrm{mg} / \mathrm{kg}$ ). Isotype control $\mathrm{IgG}$ (mouse IgG2ak, Sigma) was used as a control. One week after administration, (3 mice/group) complete blood counts and serum chemistry were analysed and dissected organs were pathologically evaluated by $\mathrm{H} \& \mathrm{E}$ staining.

\section{Tumor xenograft and antibody therapy}

Healthy female CB17/severe combined immunodeficient (SCID) mice and non-obese diabetic (NOD)/SCID mice at 6-week-old were obtained from Charles River Japan (Yokohama, Japan). Animals were maintained in a specific pathogen-free facility. For xenograft experiments, SCID and NOD/SCID mice were inoculated subcutaneously with $2 \times 10^{6}$ TE14 cells in a total volume of $100 \mu \mathrm{l}$ of $1 / 1(\mathrm{v} / \mathrm{v})$ PBS/Matrigel (BD Biosciences) into the flank. When the mean tumor sizes reached approximately $70 \mathrm{~mm}^{3}$ for TE14, mice were then randomly divided into two groups (six mice/group) and isotype control IgG (mouse IgG2aк, Sigma) or chicken/mouse chimeric anti-human GPC1 mAb (clone 1-12, Fc type is mouse $\operatorname{IgG2a}$ ) was administered at a dose of $10 \mathrm{mg} / \mathrm{kg}$ in $400 \mu \mathrm{l}$ of PBS twice-weekly for 3 weeks. Tumor sizes were measured twice-weekly using vernier callipers throughout the study. Tumor volumes were determined by measuring two dimensions, length $(L)$ and width $(W)$ and calculating the volume as $\left(W^{2} \times L\right) / 2$. Tumors were resected and weighed 32 days (TE14 cells) after first treatment. All animal experiments were conducted according to the institutional ethical guidelines for animal experimentation of the National Institute of Biomedical Innovation, Health and Nutrition.

\section{Development of patient tumor-derived xenograft (PDX) mouse models and antibody therapy}

Healthy female NOD/Shi-scid-IL2R $\gamma$ null (NOG) mice at 6-week-old were purchased from Central Institute for Experimental Animals (Kawasaki, Japan). Animals were maintained at the local animal facility according to the legislation and ethical approval was obtained for the establishment of PDX. Use of human tissues was permitted by the ethics committees of the Osaka University, Graduate School of Medicine and the National Institute of Biomedical Innovation, Health and Nutrition. The ESCC8-PDX mouse models were established with fresh ESCC tissues endoscopically resected from ESCC patients. Briefly, endoscopically resected patient's ESCC tissues (P0 tissue) were subcutaneously implanted into female NOG mice within two hours after the resection. The xenografted ESCC tumors (about $500 \mathrm{~mm}^{3}$ ) were harvested from the tumor bearing mice and were further implanted in female NOG mice for expansion. After three consecutive mouse-to-mouse passages, the xenograft was considered to be stabilized. To assess the therapeutic efficacy of anti-GPC1 mAb, tumor tissues were subcutaneously implanted into female SCID mice. When the mean tumor sizes reached approximately $70 \mathrm{~mm}^{3}$, mice were randomly divided into two groups (seven mice/group) and antibodies were administered at a dose of $10 \mathrm{mg} / \mathrm{kg}$ in $400 \mu \mathrm{l}$ of PBS twice-weekly for 4 weeks.

\section{TUNEL assay}

TUNEL assay (with DAPI nuclear counterstaining) for apoptosis was conducted using the ApopTag Fluorescein In Situ Apoptosis Detection Kit (Chemicon International, Temecula, CA, USA), according to the manufacturer's instructions. The images were acquired using a fluorescence microscope (BZ-9000; KEYENCE, Osaka, Japan).

\section{Statistical analysis}

Data are shown as mean $\pm \mathrm{SD}$ for in vitro experiments and mean \pm SEM for in vivo experiments. For comparisons among three or more groups, the values were analysed by one-way ANOVA, followed by Scheffe's test. MannWhitney $U$-test was used for significant differences in two groups. Differences were considered significant at $P<0.05$.

\section{Abbreviations}

ADCC: antibody-dependent cellular cytotoxicity; CDC: complement-dependent cytotoxicity; ESCC: Esophageal squamous cell carcinoma; FACS: fluorescence activated cell sorting; GPC1: glypican-1; NOD: nonobese diabetic; SCID: severe combined immunodeficient; SD: standard deviation; siRNA: small interfering RNA.

\section{ACKNOWLEDGMENTS}

We would like to thank Y. Kanazawa, J. Yamagishi, Y. Yamamoto and H. Matsuhisa for their secretarial assistance. 


\section{CONFLICTS OF INTEREST}

The authors declare no conflicts of interest.

\section{Authors' contributions}

Conception and design: Satoshi Serada, Tetsuji Naka; Acquisition of data: Emi Harada, Satoshi Serada, Yusuke Takahashi, Analysis and interpretation of data: Emi Harada, Satoshi Serada, Minoru Fujimoto, Yusuke Takahashi, Tsuyoshi Takahashi, Hisashi Hara, Rie Nakatsuka, Takahito Sugase, Takahiko Nishigaki, Yurina Saito, Kosuke Hiramatsu, Satoshi Nojima, Risa Mitsuo, Tomoharu Ohkawara, Eiichi Morii, Masaki Mori, Yuichiro Doki; Writing, review and/or revision of the manuscript: Emi Harada, Satoshi Serada, Tetsuji Naka, Study supervision: Tetsuji Naka

\section{REFERENCES}

1. Parkin DM, Bray F, Ferlay J, Pisani P. Global cancer statistics, 2002. CA Cancer J Clin. 2005; 55:74-108.

2. Shinoda M, Hatooka S, Mori S, Mitsudomi T. Clinical aspects of multimodality therapy for resectable locoregional esophageal cancer. Ann Thorac Cardiovasc Surg. 2006; $12: 234-241$

3. Slamon DJ, Leyland-Jones B, Shak S, Fuchs H, Paton V, Bajamonde A, Fleming T, Eiermann W, Wolter J, Pegram M, Baselga J, Norton L. Use of chemotherapy plus a monoclonal antibody against HER2 for metastatic breast cancer that overexpresses HER2. N Engl J Med. 2001; 344:783-792.

4. Hara H, Takahashi T, Serada S, Fujimoto M, Ohkawara T, Nakatsuka R, Harada E, Nishigaki T, Takahashi Y, Nojima S, Miyazaki Y, Makino T, Kurokawa Y, et al. Overexpression of glypican-1 implicates poor prognosis and their chemoresistance in oesophageal squamous cell carcinoma. Br J Cancer. 2016; 115:66-75.

5. Filmus J, Selleck SB. Glypicans: proteoglycans with a surprise. J Clin Invest. 2001; 108:497-501.

6. Matsuda K, Maruyama H, Guo F, Kleeff J, Itakura J, Matsumoto Y, Lander AD, Korc M. Glypican-1 is overexpressed in human breast cancer and modulates the mitogenic effects of multiple heparin-binding growth factors in breast cancer cells. Cancer Res. 2001; 61:5562-5569.

7. Filmus J, Capurro M, Rast J. Glypicans. Genome Biol. 2008; 9:224.

8. Downward J, Yarden Y, Mayes E, Scrace G, Totty N, Stockwell P, Ullrich A, Schlessinger J, Waterfield MD. Close similarity of epidermal growth factor receptor and v-erb-B oncogene protein sequences. Nature. 1984; 307:521-527.

9. Coussens L, Yang-Feng TL, Liao YC, Chen E, Gray A, McGrath J, Seeburg PH, Libermann TA, Schlessinger J, Francke U. Tyrosine kinase receptor with extensive homology to EGF receptor shares chromosomal location with neu oncogene. Science. 1985; 230:1132-1139.

10. Ozawa S, Ueda M, Ando N, Shimizu N, Abe O. Prognostic significance of epidermal growth factor receptor in esophageal squamous cell carcinomas. Cancer. 1989; 63:2169-2173.

11. Yano H, Shiozaki H, Kobayashi K, Yano T, Tahara H, Tamura S, Mori T. Immunohistologic detection of the epidermal growth factor receptor in human esophageal squamous cell carcinoma. Cancer. 1991; 67:91-98.

12. Enzinger PC, Mayer RJ. Esophageal cancer. N Engl J Med. 2003; 349:2241-2252.

13. Itakura Y, Sasano H, Shiga C, Furukawa Y, Shiga K, Mori S, Nagura H. Epidermal growth factor receptor overexpression in esophageal carcinoma. An immunohistochemical study correlated with clinicopathologic findings and DNA amplification. Cancer. 1994; 74:795-804.

14. Petak I, Schwab R, Orfi L, Kopper L, Keri G. Integrating molecular diagnostics into anticancer drug discovery. Nat Rev Drug Discov. 2010; 9:523-535.

15. Schlessinger J, Lax I, Lemmon M. Regulation of growth factor activation by proteoglycans: what is the role of the low affinity receptors? Cell. 1995; 83:357-360.

16. Wallace PK, Howell AL, Fanger MW. Role of Fc gamma receptors in cancer and infectious disease. J Leukoc Biol. 1994; 55:816-826.

17. Qiao D, Yang X, Meyer K, Friedl A. Glypican-1 regulates anaphase promoting complex/cyclosome substrates and cell cycle progression in endothelial cells. Mol Biol Cell. 2008; 19:2789-2801.

18. Qiao D, Meyer K, Friedl A. Glypican-1 stimulates Skp2 autoinduction loop and G1/S transition in endothelial cells. J Biol Chem. 2012; 287:5898-5909.

19. Qiao D, Meyer K, Friedl A. Glypican 1 stimulates S phase entry and DNA replication in human glioma cells and normal astrocytes. Mol Cell Biol. 2013; 33:4408-4421.

20. Qiao D, Meyer K, Mundhenke C, Drew SA, Friedl A. Heparan sulfate proteoglycans as regulators of fibroblast growth factor-2 signaling in brain endothelial cells. Specific role for glypican-1 in glioma angiogenesis. J Biol Chem. 2003; 278:16045-16053.

21. Aikawa T, Whipple CA, Lopez ME, Gunn J, Young A, Lander AD, Korc M. Glypican-1 modulates the angiogenic and metastatic potential of human and mouse cancer cells. J Clin Invest. 2008; 118:89-99.

22. Kleeff J, Ishiwata T, Kumbasar A, Friess H, Buchler MW, Lander AD, Korc M. The cell-surface heparan sulfate proteoglycan glypican-1 regulates growth factor action in pancreatic carcinoma cells and is overexpressed in human pancreatic cancer. J Clin Invest. 1998; 102:1662-1673.

23. Duan L, Hu XQ, Feng DY, Lei SY, Hu GH. GPC-1 may serve as a predictor of perineural invasion and a prognosticator of survival in pancreatic cancer. Asian $\mathrm{J}$ Surg. 2013; 36:7-12. 
24. Su G, Meyer K, Nandini CD, Qiao D, Salamat S, Friedl A. Glypican-1 is frequently overexpressed in human gliomas and enhances FGF-2 signaling in glioma cells. Am J Pathol. 2006; 168:2014-2026.

25. Yokoyama T, Enomoto T, Serada S, Morimoto A, Matsuzaki S, Ueda Y, Yoshino K, Fujita M, Kyo S, Iwahori K, Fujimoto M, Kimura T, Naka T. Plasma membrane proteomics identifies bone marrow stromal antigen
2 as a potential therapeutic target in endometrial cancer. Int $\mathrm{J}$ Cancer. 2013; 132:472-484.

26. Iwahori K, Serada S, Fujimoto M, Ripley B, Nomura S, Mizuguchi H, Shimada K, Takahashi T, Kawase I, Kishimoto T, Naka T. SOCS-1 gene delivery cooperates with cisplatin plus pemetrexed to exhibit preclinical antitumor activity against malignant pleural mesothelioma. Int J Cancer. 2013; 132:459-471. 